\title{
Entdeckung von drei neuen Planeten (245), (246) und (247).
}

Planet (245). Las Berl. Circ. 246 enthält folgende Mittheilung:

"Nach brieficher Mittheilung des Herrn Director Pogson in Madras hat derselbe folgenden Planeten, welcher anfänglich für $(42)$ Isis gehalten wurde, beobachtet:

\section{Mittl. Ortszeit}

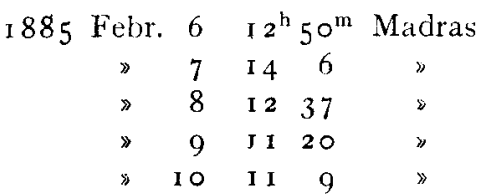

\begin{tabular}{|c|c|c|}
\hline$\alpha$ app. & $\delta$ app. & $\mathrm{Gr}$ \\
\hline $6^{\mathrm{h}} 45^{\mathrm{m}} 30^{\mathrm{s}}$ & $+28^{\circ} 32: 9$ & \\
\hline $\begin{array}{lll}6 & 45 & 3\end{array}$ & +2832.9 & \\
\hline $644 \quad 40$ & +2833.0 & \\
\hline $\begin{array}{lll}6 & 44 & \text { I } 8\end{array}$ & +2832.6 & \\
\hline $6435^{8}$ & +2832.5 & \\
\hline
\end{tabular}

Eine am 8. März von der Centralstelle an die Sternwarte in Madras ergangene Aufforderung, eine neuere Beobachtung telegraphisch einzusenden, wurde am I6. März Abends folgendermaassen beantwortet:

Unspent twenty position unwell estationary February hickory censer appropriatly cantilivere condenc marsh crawfish cetacean apt mix desideratum. Pogson, Observatory Madras.

Nach Verbesserung einiger leicht ersichtlichen Verstümmelungen lautet das Telegramm:

"Your telegram has been received. Twenty positions will be sent as soon as possible.

(Stationary) Febr. 22.2830 Gr. M. T. AR. $=6^{\mathrm{h}} 4 \mathrm{I}^{\mathrm{m}} 52^{\mathrm{s}} .9$ PD. $=6 \mathrm{I}^{\circ} 33^{\prime} 4^{\prime \prime}$

March $13.25^{2} \gg 646$ I $8.9 \quad 6$ I 5514 《.

Inzwischen ist der Planet am 9. März auf der Wiener Sternwarte von Dr. J. Palisa aufgefunden und beobachtet worden. Seine erste telegraphische Nachricht vom 10. März, in Rectascension um I z" abweichend von einer späteren Mittheilung vom I I. März, ist sofort an die Mitglieder der Centralstelle weiter befördert worden. Die hier bis jetzt eingegangenen Beobachtungen von Dr. J. Palisa lauten:

r 885 März $99^{\mathrm{h}} 34^{\mathrm{m}} 7^{\mathrm{s}}$ M.Z. Wien $\alpha=6^{\mathrm{h}} 44^{\mathrm{m}} 42^{\mathrm{s}} \cdot 49(9.349) \quad \delta=+28^{\circ} 10^{\prime} \quad 0^{\prime \prime} 5\left(0.5^{2} 8\right)$ Gr. I $2^{\mathrm{m}} \cdot 5$ Telegramm und Brief.
- 1075527
$\begin{array}{llll}6 & 45 & 3.78 & (8.683)\end{array}$
$+28845.3(0.480)$

Planet (246). Die Centralstelle erhielt am 7. März Nachmittags von der Sternwarte Paris das folgende Telegramm:

Planète Borrelly 6 mars $9^{\mathrm{h}} 7^{\mathrm{m}} \mathrm{I}$ t. m. Marseille ${ }^{*}$ )

AR. app. $=166^{\circ} 33^{\prime} 23^{\prime \prime}$ DP. app. $=82^{\circ} 50^{\prime} 43^{\prime \prime}$ mouv. diurne: - $12^{\prime}$ et $-9^{\prime}$ grandeur II.I $2^{m}$.

Das Telegramm wurde sofort weiter befördert.

Dr. S. Oppenheim theilt die folgende am 6 Zöller der Wiener Sternwarte angestellte Beobachtung mit:

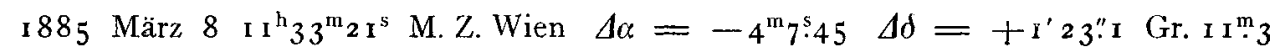
$a$ app. $=\mathrm{II}^{\mathrm{h}} 4^{\mathrm{m}} 38^{\mathrm{s}} 86\left(8.620_{\mathrm{n}}\right) \quad \delta$ app. $=+7^{\circ} 3 \mathrm{I}^{\prime} 5 \mathrm{I}^{\prime \prime} \circ(0.759)$

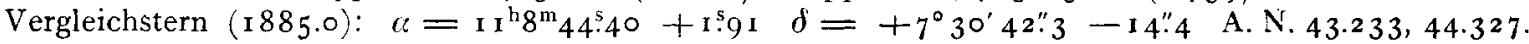

*) Nach den Comptes Rendus Nr. 10 lautet diese Beobachtung, etwas abweichend:

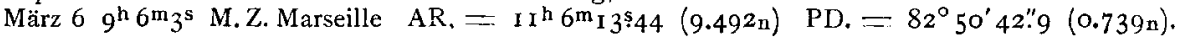

Planet (247). Am 15. März Mittags ging von der Sternwarte Düsseldorf folgendes Telegramm ein:

Planet Luther März 14 I I $^{\mathrm{h}} \mathbf{1} 7^{\mathrm{m}} \cdot 8$ M. Z. Düsseldorf

AR. app. $=177^{\circ} \mathbf{1 2}^{\prime}$ PD. app. $=84^{\circ} 47^{\prime}$ Grösse $\mathrm{I}^{\mathrm{m}}$. Trübe.

Luther.

Die Entdeckung wurde sofort den Mitgliedern der Centralstelle telegraphisch mitgetheilt.

Kiel I 885 März I 6 .

Kr.

Berichtigung zu Nr. 2653 Bd, 1 I I pag. 201 und 202. Auf dieser Seite sind während des Reindrucks die Columnen der Tabelle in störender Weise verschoben worden, wofür der Leser um Entschuldigung gebeten wird.

\section{In halt:}

Zu Nr. 2654-55. V. Knorre. Beobachtungen von Cometen und kleinen Planeten, 209. - G. Cacciatore. Passaggi della Macchia Rossa di Giove per il meridiano centrale. 227. - C. Schultz-Steinheil. Beobachtungen des Cometen 1884 III (Wolf). 229. - C. H. F. Peters. Beobachtungen des Cometen I884 III (Wolf). 23I. - H. V. Eggbert. Comparison stars for Comet I884 III (Wolf). 233. 7. Lamp. Beobachtungen des Cometen I884 III (Wolf). 235. - E. Lamp. Ephemeride des Cometen I884 III (Wolf). 235. Vermischte Nachrichten. 237. - Entdeckung von drei neuen Planeten (245), (246) und (247). 239. - Berichtigung. 239.

Geschlossen $188_{5}$ März 17. Herausgeber: A. Krueger. Druck von C. F. Mohr. Expedition: Sternwarte in Kiel. 